\title{
1 A conservation law for virus infection kinetics in vitro
}

2

3 Yusuke Kakizoe ${ }^{1}$, Satoru Morita ${ }^{2}$, Shinji Nakaoka ${ }^{3}$, Yasuhiro Takeuchi ${ }^{4}$, Kei Sato ${ }^{5,6}$,

4 Tomoyuki Miura ${ }^{5}$, Catherine A. A. Beauchemin ${ }^{7}$, and Shingo Iwami ${ }^{1,6,8, \uparrow}$

5

6

${ }^{1}$ Department of Biology, Kyushu University, Fukuoka, Japan

${ }^{2}$ Department of Mathematical and Systems Engineering, Shizuoka University, Shizuoka, Japan

${ }^{3}$ Graduate School of Medicine, University of Tokyo, Tokyo, Japan

${ }^{4}$ College of Science and Engineering, Aoyama Gakuin University, Kanagawa, Japan

${ }^{5}$ Institute for Virus Research, Kyoto University, Kyoto, Japan

${ }^{6}$ CREST, JST, Saitama, Japan

${ }^{7}$ Department of Physics, Ryerson University, Toronto, Canada

${ }^{8}$ PRESTO, JST, Saitama, Japan

${ }^{\dagger}$ E-mail: siwami@kyushu-u.org

Abstract. Conservation laws are among the most important properties of a physical system, but are not commonplace in biology. We derived a conservation law from the basic model for viral infections which consists in a small set of ordinary differential equations. We challenged the conservation law experimentally for the case of a virus infection in a cell culture. We found that the derived, conserved quantity remained almost constant throughout the infection period, implying that the derived conservation law holds in this biological system. We also suggest a potential use for the conservation law in evaluating the accuracy of experimental measurements.

Keywords: Mathematical model, Conservation law, Population dynamics, Virus infection 


\section{Introduction}

In physics, a conserved quantity of a dynamical system is a quantity expressed as a function of the systems' dynamical, dependent variables, but which remains constant along each trajectory of the system. In the natural sciences, various conservation laws, such as the conservation of mass-energy and of linear momentum, have been identified and experimentally validated (Blanchard et al., 2005). Conservation laws for generalized symmetric 2 n-dimensional nonlinear systems of differential equations were also more recently derived and analyzed (Uechi and Akutsu, 2012; Uechi and Akutsu, 2013), but with limited or no experimental validation. In theoretical biology, such equation systems, as well as larger sets of differential equations, are often used to describe complex biological phenomena. Two good examples are the Lotka-Volterra model and the Kermack-McKendrick models, which are almost identical sets of equations but are used to describe two different dynamical systems. The Lotka-Volterra model is well-studied in ecology (Lotka, 1956), and describes the interactions and resulting dynamics of a population of prey, $X(t)$, and predators, $Y(t)$ over time, $t$, as

$$
\frac{d X(t)}{d t}=r X(t)-a X(t) Y(t), \quad \frac{d Y(t)}{d t}=b X(t) Y(t)-c Y(t) .
$$

41 From these two equations, we obtain the relation

$$
\frac{d X(t)}{d t}\left(b-\frac{c}{X(t)}\right)+\frac{d Y(t)}{d t}\left(a-\frac{r}{Y(t)}\right)=0 .
$$

42 Integrating both sides yields the conserved quantity $V(X(t), Y(t))=b X(t)-c \ln X(t)+$ $43 a Y(t)-r \ln Y(t)$, which is known as a Lyapunov function of the model. Similarly, the 44 Kermack-McKendrick model (Kermack and McKendrick, 1927), which is almost identical to the Lokta-Volterra model, is used in epidemiology to describe the transmission of an infectious disease by infected individuals, $I(t)$, to susceptible individuals, $S(t)$, over time, $t$, as

$$
\frac{d S(t)}{d t}=-\beta S(t) I(t)-\mu S(t), \quad \frac{d I(t)}{d t}=\beta S(t) I(t)-a I(t) .
$$

Using a calculation similar to that shown above for the Lokta-Volterra model, one finds the following conserved quantity for the Kermack-McKendrick model: $W(S(t), I(t))=\beta S(t)-$ $a \ln S(t)+\beta I(t)+\mu \ln I(t)$. From this particular conserved quantity, an important concept in theoretical epidemiology called "final size equation" was derived (Anderson, 1991). This suggests that conservation laws likely exist in quantitative descriptions of the population dynamics of real biological systems. However, although the concept of conservation laws and the conserved quantities themselves can provide important insights in biology, direct evidence of such laws in biological systems is currently lacking, and no method has been established to prove otherwise (Hagiwara et al., 2011). 
57 cell biology has provided many new insights into the complex interactions between cells, 58 proteins, and genes. Similarly, in virology, this knowledge enables us to track the kinetics of 59 virus and target cells in experiments, especially in cell cultures, thus generating a time-course of 60 experimental data. To better understand the quantitative features of virus replication, we have 61 developed an experimental-mathematical system that quantifies the kinetics of virus infection, 62 combining time-course experimental data with mathematical models (Fukuhara et al., 2013; 63 Iwami et al., 2012a; Iwami et al., 2012b). Here, for the standard, basic model of viral infection 64 kinetics widely used to analyze viral kinetics in vivo and in vitro (Nowak and May, 2000), we 65 mathematically derive a conserved quantity and provide experimental confirmation of its 66 conservation in our in vitro virus infection assays. To our knowledge, this is the first time that 67 the existence of a conservation law for population dynamics in a real biological system has been 68 experimentally confirmed. 


\section{Results and Discussion} \\ 2.1. Derivation of a conservation law for viral infections}

We introduce the well-known, extensively used basic model to describe virus infection

kinetics (Nowak and May, 2000; Perelson, 2002):

$\frac{d T(t)}{d t}=-\beta T(t) V(t), \quad \frac{d I(t)}{d t}=\beta T(t) V(t)-\delta I(t), \quad \frac{d V(t)}{d t}=p I(t)-c V(t)$,

73 where $T(t)$ and $I(t)$ are the numbers of target (susceptible) and infected (virus-producing)

74 cells per $\mathrm{ml}$ of medium, respectively, and $V(t)$ is the viral load per $\mathrm{ml}$ of medium. Parameter

$75 \delta$ represents the death rate of infected cells, $c$ is the rate of virus loss, $\beta$ is the rate constant

76 for infection of target cells by virus, and $p$ is the virus production rate per cell. In our

77 experiments discussed below, the viral load was measured either as a total count of extracellular

78 virions, expressed in units of RNA copies/ml (two RNA copy equals one virion) and measured

79 via quantitative PCR, or as a relative concentration of extracellular infectious virions, expressed

80 in units of $\mathrm{TCID}_{50} / \mathrm{ml}$ (proportional to the number of infectious virions) and measured via virus

81 titration on cell cultures (see Materials and methods for details). For each of the daily

82 measurements of the virus concentration, the medium in our experiments was harvested,

83 reducing the viral concentration by $85.4 \%$. This removal can be captured using Eq. (1) by

84 approximating the punctual removal of virus at each sampling time as a continuous, exponential

85 decay of the viral load over the period between samples (one day). In such a case, parameter $c$

86 corresponds to the sum of the rate of virus loss due to harvesting of the medium plus the rate of

87 loss due to degradation of either the total extracellular virus (when $V(t)$ is measured in units of

88 RNA copies $/ \mathrm{ml}$ ) or of infectivity (when $V(t)$ is measured in units of $\mathrm{TCID}_{50} / \mathrm{ml}$ ). In our earlier

89 work, we have shown that approximating punctual removal as a continuous exponential decay

90 has minimal impact on the model parameters and provides an appropriate fit to the experimental

91 data (Iwami et al., 2012a; Iwami et al., 2012b), and we will be using this approximation here.

92 For completeness, in Appendix 5.1., we also consider a model analogous to (1) wherein

93 punctual removal has been included by using impulsive differential equations, and to which we

94 will refer as the punctual removal model. Note that the measurement units of virus (RNA copies

95 or $\mathrm{TCID}_{50}$ ) used in our analysis changes both the units and physical meaning of the virus

96 production $(p)$ and cell infection $(\beta)$ rates, changes the meaning and value of the virus loss $(c)$,

97 and the value of the infectious cell lifespan estimated from the experimental data $(\delta)$.

98 Let us define a new variable, $L(t) \equiv \ln [T(t) / N]$, where $N$ is the total cell 99 concentration at time $t=0$, i.e., $N=T(0)+I(0)$, and is used to make the argument of the $\ln$ 100 function unitless. It follows from $d T(t) / d t$ in Eq.(1) that $d L(t) / d t=-\beta V(t)$. Then from 101 Eq.(1) we have 


$$
\frac{d T(t)}{d t}+\frac{d I(t)}{d t}+\frac{\delta}{p} \frac{d V(t)}{d t}-\frac{\delta c}{p \beta} \frac{d L(t)}{d t}=0,
$$

102 and integrating both sides of Eq.(2), we obtain

$$
H=T(t)+I(t)+\frac{\delta}{p} V(t)-\frac{\delta c}{p \beta} L(t) .
$$

103 This equation holds for all $t$, indicating that $H$ is a conserved quantity of the dynamical 104 system in Eq.(1). Note that it has been shown in prior work that the more biologically realistic 105 inclusion of an eclipse phase into Eq.(1) is required to correctly capture infection kinetics, and 106 that the duration of this phase is best captured by a Gaussian-like distribution (normal, 107 log-normal, gamma, Erlang, etc.), but is incorrectly captured by an exponential or Dirac-delta 108 distribution (Holder and Beauchemin, 2011; Petravic et al., 2014; Pinilla et al., 2012). In 109 Appendix 5.2., we derive quantity $H$ for a model analogous to (1) wherein an 110 Erlang-distributed eclipse phase has been included. We show that one arrives at what is 111 essentially the same expression for $H$ with an additional term for eclipse cells, $+E(t)$, such 112 that expression (3) also holds under this more realistic model. However, the conservation law 113 does not hold if cell regeneration and cell division is non-negligible or if the lifespan of 114 infectious cells follows a Gaussian-like distribution rather than the exponential distribution used 115 here (not shown).

\subsection{Biological interpretation of the conservation law for viral infection}

Through simple re-arrangement of conserved quantity $H$, we can arrive at another conserved quantity, $Q$, with a clearer and more useful biological interpretation. Because Eq.(3) holds at $t=0$, we have the following relation

$$
T(t)+I(t)+\frac{\delta}{p} V(t)-\frac{\delta c}{p \beta} L(t)=T(0)+I(0)+\frac{\delta}{p} V(0)-\frac{\delta c}{p \beta} L(0) .
$$

In typical cell culture experiments, infection is initiated from a virus inoculum in a fully susceptible cell population such that $I(0) \approx 0$. Furthermore, since $L(t) \equiv \ln [T(t) / N]$, we have $L(0)=0$ such that Eq.(4) becomes

$$
T(0)=T(t)+I(t)+\frac{\delta}{p}[V(t)-V(0)]-\frac{\delta c}{p \beta} L(t) .
$$

124 We remark that $L(t)=-\beta \int_{0}^{t} V(s) \mathrm{d} s$, and re-write Eq.(5) as

$$
T(0)=T(t)+I(t)+\frac{V(t)-V(0)}{(p / \delta)}+\frac{c \int_{0}^{t} V(s) \mathrm{d} s}{(p / \delta)} .
$$

125 Note that the term $p / \delta$ corresponds to the viral burst size which, depending on the measurement units of the viral load, corresponds to either the number virus RNA copies or 
such, the term $[V(t)-V(0)] /(p / \delta)$ essentially converts the observed total or infectious virus concentration in the medium at time $t$ into the concentration of cells that would have had to die to produce them. The term $c \int_{0}^{t} V(s) \mathrm{d} s /(p / \delta)$ effects the same conversion of virus into a cell count, but does so for the sum total of all virus (RNA copies) or only the infectious virus $\left(\mathrm{TCID}_{50}\right)$ which are no longer present in the medium because they have been harvested and degraded at rate $c$ from time $t=0$ up to and including time $t$. In other words, the terms $[V(t)-V(0)] /(p / \delta)+c \int_{0}^{t} V(s) \mathrm{d} s /(p / \delta)$ correspond to the total concentration of cells which have died due to infection by time $t$, i.e., $D(t)=\int_{0}^{t} \delta I(s) \mathrm{d} s$. In the case of a non-lytic virus (i.e., $\delta=0$ ), the burst size becomes infinity, which implies these two terms become 0 and Eq. (6) becomes simply $T(0)=T(t)+I(t)$. Thus, for all $t$, the total mass (or energy) of the system, which is contained in the initial target cells, $T(0)$, remains nearly constant over time in an isolated system even as cells change state $(T \rightarrow I)$ or are converted to total or infectious virions $(I \rightarrow V)$. For convenience, from here on, we will instead make use of the following normalized, conserved quantity

$$
Q=1=\frac{1}{T(0)}\left\{T(t)+I(t)+\frac{V(t)-V(0)}{(p / \delta)}+\frac{c \int_{0}^{t} V(s) d s}{(p / \delta)}\right\}
$$

\subsection{Experimental confirmation of the conservation law for viral infection}

We can verify whether this theoretical conservation law holds experimentally by calculating the normalized quantity $Q$ in Eq.(7) over the course of an experimental viral infection in a cell culture. We infected HSC-F cell cultures with two different strains of the simian-human immunodeficiency virus (SHIV-KS661 and SHIV-\#64 (Kozyrev et al., 2001): the intravenous infection of rhesus macaques with SHIV-KS661 consistently results in high viremia and CD4+ $\mathrm{T}$ cell depletion, whereas infection with SHIV-\#64 causes more subdued pathogenesis), as previously described (Iwami et al., 2012a; Iwami et al., 2012b), with additional details in Materials and methods. Briefly, cultures of HSC-F cells were infected with SHIV-KS661 (or SHIV-\#64) at $24 \mathrm{~h}$ prior to the first measurement (i.e., $t=-24 \mathrm{~h}$ ), incubated with the virus for $4 \mathrm{~h}$ (i.e., the cell were placed in fresh, virus-free medium at time $t=-20 \mathrm{~h}$ ), and sampled daily over 10 consecutive days (i.e., $t=0,1, \ldots, 9$ days). Each day, most of the virus $(85.4 \%)$ and a small percentage of the cells $(5.5 \%)$ were removed from the culture supernatant for measurement, and fresh medium was added. Each sample was assayed to determine the concentration of cells (cells $/ \mathrm{ml}$ ) and the fraction of these cells which had either remained uninfected $($ Nef-negative $=$ Target, $T(t)$ ) or had been infected (Nef-positive = Infected, $I(t)$ ) by SHIV, and to determine the total viral load (RNA copies/ml) and infectious viral titer $\left(\mathrm{TCID}_{50} / \mathrm{ml}\right)$ of SHIV-KS661 (or SHIV-\#64) in the extracellular medium. The infection experiments were performed at two different values of the initial viral inoculum 
162 (multiplicity of infection (MOI) of $2.0 \times 10^{-4}$ and $2.0 \times 10^{-5}$ ) so as to provide sufficient 163 data for robust parameter estimation and for calculation of $Q$.

164 To determine the parameter values and initial conditions in Eq.(1) for SHIV-KS661 165 and SHIV-\#64 infections, we fitted Eq.(1) to the time-course data listed under "For parameter 166 estimation $\left(2.0 \times 10^{-4} \mathrm{MOI}\right) "$ in Table 1 (see Materials and methods). The parameter and 167 initial values estimated by fitting Eq.(1) to datasets using either the total viral load (RNA 168 count $/ \mathrm{ml})$ or the infectious viral titer $\left(\mathrm{TCID}_{50} / \mathrm{ml}\right)$ concentrations are listed in Tables 2 and $\mathbf{3}$, 169 with the model's predicted kinetics for these best-fit parameter estimates presented alongside the 170 data in Figures 1(A)(B) and 2(A)(B), respectively. For the alternate model with punctual virus 171 removal at each sampling time (Appendix 5.1.), the estimated parameters and initial values are 172 listed in Tables $\mathbf{4}$ and 5, with their predicted kinetics presented alongside the data in Figures $173 \mathbf{3}(\mathbf{E})(\mathbf{F})$ and $\mathbf{4}(\mathbf{E})(\mathbf{F})$, respectively.

174 The degradation rate of total virus (RNA copies) is inferior (slower) than that of 175 infectious virus $\left(\mathrm{TCID}_{50}\right)$. For example, for SHIV-KS661, we have determined in previous work 176 that the degradation rate of total extracellular virus (RNA copies) is $0.039 /$ day, whereas that of 177 infectious virus $\left(\mathrm{TCID}_{50}\right)$ is $0.91 /$ day (Iwami et al., 2012a; Iwami et al., 2012b). Since 178 infectious virus degrades at a higher rate than total virus (which includes both infectious and 179 non-infectious virus), we expect that Eq.(1) should be appropriate when $V(t)$ is proportional to 180 infectious virus $\left(\mathrm{TCID}_{50}\right)$. But when $V(t)$ represents the total virus (RNA copies), a more 181 accurate model might require that, at the very least, the infection rate, $\beta$, decrease over time, $182 \beta(t)$, to reflect that for an equivalent number of total virions, one has a decreasing number of 183 infectious virions (or decreasing infectivity) as time passes. By using the same model, Eq. (1), 184 to fit both the total (RNA copies) and infectious $\left(\mathrm{TCID}_{50}\right)$ viral load, we are assuming that the 185 ratio of infectious to total viral load is relatively constant over the course of our infection. This 186 can be justified in part because, during the viral load growth phase (virus upslope), when the 187 majority of cell infection takes place, the rate of virus production exceeds the rate of virus 188 clearance such that the ratio of infectious to total virus is roughly constant. Additionally, in our 189 experiments, the rate of virus loss due to harvesting $(-\ln (1-0.854) /(1$ day $)=1.92 /$ day $)$ 190 exceeds the rate of degradation of either extracellular viral RNA copies (e.g., 0.91/day) or 191 infectious, $\mathrm{TCID}_{50}$ viral titer (e.g., 0.039/day), such that it dominates the rate of viral loss, and 192 further helps maintain the ratio of infectious to total virus relatively constant over the course of 193 the infection. While it is clear from Table 1 that the ratio of $\mathrm{TCID}_{50} / \mathrm{RNA}$ in our experiments 194 varies significantly for infection with SHIV-KS661 at an MOI of $2.0 \times 10^{-4}$, it does not 195 decrease too significantly up to viral titer peak (day 6) in the other three infection time courses. 196 This suggests that the large deviation in this one infection time course is more likely a stochastic 197 deviation due to experimental error rather than a significant trend, and the use of Eq. (1) to 
198 capture our infection experiments measuring either total total (RNA copies) or infectious

\section{$\left(\mathrm{TCID}_{50}\right)$ viral load is appropriate, though it will contribute some error.}

As evident from these figures, Eq.(1) accurately models these cell culture experiments, although there is some difference between our prediction and experimental data caused by the property of Eq.(1) that target cells are partially depleted, and will not ultimately approach "0".

Using the experimental data, the theoretically conserved quantity $Q$ was determined by substituting the parameter values in Tables 2 and $\mathbf{3}$ into Eq.(7). In this calculation, the time-course data listed under "For calculation of $Q\left(2.0 \times 10^{-5} \mathrm{MOI}\right)$ " in Table 1 were used. The data at day 0 were assumed as $T(0)$ and $\int_{0}^{t} V(s) \mathrm{d} s$ was calculated from the viral load data using the trapezoidal rule. In Materials and methods, we provide an example for the calculation of $Q$ at one time point. The calculated $Q \mathrm{~s}$, based on datasets including the viral RNA and infectivity, at each time point are plotted in Figure 1(C)(D) and 2(C)(D), respectively. For the punctual removal model, the calculated $Q$ s are also plotted in Figures $3(\mathbf{G})(\mathbf{H})$ and $\mathbf{4}(\mathbf{G})(\mathbf{H})$, respectively.

Theoretically, $Q$ should remain around 1 throughout the virus infection if the model is a valid representation of the infection kinetics and if the conservation law holds. Interestingly, the calculated $Q$ values remain around 1, with slight fluctuations, throughout the infection period for both virus strains. This provides compelling evidence that the mathematical model used is an appropriate description of the system and that, as such, its associated conservation law does hold over the course of experimental viral infections in cell cultures, at least for the SHIV-KS661 and SHIV-\#64 infection of HSC-F cells. Conversely, this means that the conservation law can be used as a measure of the degree of uncertainty and variability in experimental measurements, namely by comparing the standard deviations (SDs) of our computed experimental $Q$ values from the true, theoretical value of $Q=1$. In our experiments, we measured either the total viral load via quantitative RT-PCR or the infectious viral titer via $\mathrm{TCID}_{50}$ assays performed in HSC-F cell cultures, and obtained higher SDs for $Q$ values based on the $\mathrm{TCID}_{50}$ than for those based on RT-PCR (Tables 6 and 7). This is consistent with the known, greater experimental measurement errors and poorer sensitivity (larger limit of detection) of $\mathrm{TCID}_{50}$ assays compared to RT-PCR assays (see the numerous d.1. entries in Table 1). While the computed $Q$ values depend on both the experimental measurements and the estimated parameters, we have determined through a parameter sensitivity analysis (not shown) that in our experiments, deviations from $Q=1$ are largely attributable to experimental measurement errors rather than error in parameter estimates. As discussed in (Fukuhara et al., 2013; Iwami et al., 2012a; Iwami et al., 2012b), our experimental-mathematical strategy can robustly estimate parameter values from time-course data, even if there are relatively greater errors in viral load, because Eq.(1) is fitted simultaneously to the concentrations of target cells, 
234 infected cells and the viral load, which complement one another, minimizing the impact of 235 stochastic deviations present in any one particular data set. Thus, the higher SDs in the $Q$ 236 values estimated from the viral load measured via the $\mathrm{TCID}_{50}$ assay reflects larger measurement 237 errors in this assay, rather than errors in parameter estimation. 


\section{3. Conclusion}

239 Although nonlinear phenomena in virus infection experiments are usually difficult to 240 understand and explain intuitively, the dynamical behavior of the system can be exposed 241 through well-constructed mathematical models. The experimental-mathematical strategy is a 242 powerful methodology used to quantitatively investigate the dynamics of virus infections that is 243 not accessible by conventional experimental strategies (Fukuhara et al., 2013; Iwami et al., 244 2012a; Iwami et al., 2012b). To date, the basic model has been empirically used as a de facto 245 standard model for quantitatively understanding nonlinear phenomena, especially in cell 246 cultures experimentally infected with virus, which are considered as closed systems 247 (Beauchemin et al., 2008; Fukuhara et al., 2013; Iwami et al., 2012a; Iwami et al., 2012b;

248 Mohler et al., 2005; Pinilla et al., 2012). In this work, we have derived a conserved quantity 249 with a clear biological interpretation and have shown that our conservation law holds in our cell 250 culture experiments. Furthermore, we showed that computation of our conserved quantity from 251 experimental data can serve as a valuable measure of the degree of experimental error, and 252 could be used as a data validation tool to identify aberrant or erroneous measurements. Although 253 the conservation law was shown here to hold only for two different strains of SHIV, it should 254 hold for all other lytic virus in cultures where cell division is negligible over the time scale of 255 the infection experiment. 


\section{Materials and methods}

\subsection{SHIV infection in HSC-F cell culture}

The virus solution of SHIV-KS661 (Shinohara et al., 1999) (or SHIV-\#64 (Kozyrev et al., 2001)) was prepared in a CD4 ${ }^{+}$human T lymphoid cell line, M8166 (a subclone of C8166) (Clapham et al., 1987), and was stored in liquid nitrogen until use. The HSC-F cell line (Akari et al., 1996) was cultured in a culture medium (RPMI-1640 supplemented with $10 \%$ fetal calf serum) at $37^{\circ} \mathrm{C}$ and $5 \% \mathrm{CO}_{2}$ in humidified conditions.

Each experiment was performed using 2 wells of a 24-well plate with a total suspension volume of $2 \mathrm{ml}(1 \mathrm{ml}$ per well $)$ and an initial cell concentration of $T_{0}=6.46 \times 10^{6}$ cells $/ \mathrm{ml}$ in each well. Because the initial cell concentration is close to the carrying capacity of 24-well plates, and HSC-F cells replicate slowly, and in the absence of SHIV-KS661 (or SHIV-\#64) infection, the population of target cells changes very little on the timescale of our experiment (data not shown). We therefore neglected the effects of potential regeneration of HSC-F cells in our analysis and in constructing the mathematical model.

For virus infection, cultures of HSC-F cells were inoculated $24 \mathrm{~h}$ prior to the first infection sampling $(t=-24 \mathrm{~h})$ at two different multiplicity of infections (MOIs) of $2.0 \times$ $10^{-4}$ or $2.0 \times 10^{-5} \mathrm{TCID}_{50}(50 \%$ tissue culture infectious dose) per cell of SHIV-KS661 (or SHIV-\#64), and were incubated at $37^{\circ} \mathrm{C}$. Four hours after inoculation $(t=-20 \mathrm{~h})$, the cells were washed to remove the remaining viruses and were replaced into a fresh culture medium. The culture supernatant was harvested daily for 10 days $(t=0,1, \ldots, 9 \mathrm{~d})$, and was replaced with fresh medium. On a daily basis, $5.5 \%$ of the cells in the culture were harvested to measure the number of target cells and infected cells. Cells were counted by staining them with an anti-SIV Nef monoclonal antibody (04-001, Santa Cruz Biotechnology, Santa Cruz, CA) labeled by Zenon Alexa Fluor 488 (Invitrogen, Carlsbad, CA), as previously described (Iwami et al., 2012a; Iwami et al., 2012b). Each harvested supernatant, including $85.4 \%$ of the culture virus was stored at $-80^{\circ} \mathrm{C}$, and the amount of viral RNA was quantified by RT-PCR, as previously described (Iwami et al., 2012a; Iwami et al., 2012b; Motohara et al., 2006). The infectious viral load was measured by $\mathrm{TCID}_{50}$ assay in HFC-S cell cultures using 96-well flat bottom plates at cell concentrations of $1.0 \times 10^{6}$ cells $/ \mathrm{ml}$. The titer of the virus was determined as described by Reed and Muench (Reed and Muench, 1938). The time-course data of this experiment are summarized in Table 1.

\subsection{Extraction of parameters from the experimental infection data}

290

In relating our daily experimental measurements at time $t=0,1, \ldots, 9$ day to our mathematical models, we define $t=0$ as the time of our first experimental measurements, i.e. when $T(0), I(0)$, and $V(0)$ are measured. As such, by time $t=0$, some cells $(T)$ have 
292 become infected $(I)$, and while the inoculum virus has been rinsed, some new virus $(V)$ will have been produced by the newly infected cells. Parameter $c$ in Eq.(1), or $c_{\text {deg }}$ in the punctual 294 removal model, were held fixed at their value estimated in previous work (Iwami et al., 2012a; 295 Iwami et al., 2012b). The concentration of uninfected target cells at measurement time $t=0$, $296 T(0)$, was fixed to its experimentally measured quantity. The remaining two concentrations, $297 I(0)$, and $V(0)$, had to be estimated through fitting because they were below detection levels at 298 that measurement time. Note that while $V(0)$ was above the detection limit when measured in 299 units of RNA copies/ml, we still estimated its value through fitting so as to use the same analysis performed when virus was in units of $\mathrm{TCID}_{50} / \mathrm{ml}$.

Eq.(1) (or the punctual removal model) was fitted simultaneously to the $\log _{10}$ of the concentrations of target cells, infected cells, and of either total (RNA) or infectious $\left(\mathrm{TCID}_{50}\right)$ virus by nonlinear least-squares regression using the FindMinimum package of Mathematica 9.0 which minimizes the sum of squared residuals (SSR). Experimental measurements below the detection limit were excluded from the SSR calculation.

\subsection{Computation of conserved quantity $Q$ from experimental measures}

inoculation using the experimental viral RNA data (the shaded values in Table 1) as follows

$$
Q=\frac{1}{T(0)}\left\{T(5)+I(5)+\frac{V(5)-V(0)}{(p / \delta)}+\frac{c \int_{0}^{5} V(s) d s}{(p / \delta)}\right\}
$$

310 From fitting Eq.(1) to the experimental data, we obtained estimated values for $p=3.2 \times 10^{4}$ 311 (RNA copies/cell) $\cdot$ day $^{-1}, \delta=1.71$ day $^{-1}$ and $c=2.02$ day $^{-1}$ (see Table 2), which we use in 312 the above equation to compute $Q$. Note that both the degradation rate of viral RNA and the $31385.4 \%$ removal virus due to experimental sampling are included in the value for the clearance 314 rate, $c$, by approximating the decrease of virus as a continuous exponential decay. The loss of $3155.5 \%$ of cell at each sampling point due to the harvesting of the sample is assumed to be 316 negligible. The value of the integral $\int_{0}^{5} V(s) d s$ was approximated using the trapezoidal rule in $317 \quad \log$-scale, namely

$$
\begin{aligned}
\int_{0}^{5} V(s) d s \approx[ & 10^{\left(\log _{10} V(0)+\log _{10} V(1)\right) \times[1 \text { day }] / 2}+10^{\left(\log _{10} V(1)+\log _{10} V(2)\right) \times[1 \text { day }] / 2} \\
+ & 10^{\left(\log _{10} V(2)+\log _{10} V(3)\right) \times[1 \text { day }] / 2}+10^{\left(\log _{10} V(3)+\log _{10} V(4)\right) \times[1 \text { day }] / 2} \\
+ & \left.10^{\left(\log _{10} V(4)+\log _{10} V(5)\right) \times[1 \text { day }] / 2}\right]=1.47 \times 10^{9} \mathrm{RNA} \text { copies } / \mathrm{ml} \cdot \text { day. }
\end{aligned}
$$

318 We also considered the use of an interpolation function running through the viral load data, 
319 instead of using the actual data points, to estimate this integral and obtained instead $1.16 \times 10^{9}$

320 RNA copies/ml-day. From Table 1, since $T(0)=6.31 \times 10^{6}$ cells $/ \mathrm{ml}, V(0)=1.64 \times 10^{4}$

321 RNA copies $/ \mathrm{ml}, \quad T(5)=5.91 \times 10^{6}$ cells $/ \mathrm{ml}, \quad I(5)=4.12 \times 10^{5} \mathrm{cells} / \mathrm{ml}, \quad$ and $V(5)=$ $3222.18 \times 10^{9}$ RNA copies/ml, quantity $Q$ on day 5 is given by

$\frac{1}{6.31 \times 10^{6}}\left\{5.91 \times 10^{6}+4.12 \times 10^{5}+\frac{2.18 \times 10^{9}-1.64 \times 10^{4}}{\left(3.2 \times 10^{4} / 1.71\right)}+\frac{2.02 \times 1.47 \times 10^{9}}{\left(3.2 \times 10^{4} / 1.71\right)}\right\}=1.043$.

323 For the estimated integration value using the interpolation function, quantity $Q=1.038$, which

324 shows that the trapezoidal rule provides a reasonable approximation for the integration. 


\section{Appendix}

\subsection{A punctual removal model for virus infections}

We consider punctual removal of virus due to experimental sampling by adding Dirac

328 delta function to Eq.(1) as follows:

$$
\begin{gathered}
\frac{d T(t)}{d t}=-\beta T(t) V(t), \\
\frac{d I(t)}{d t}=\beta T(t) V(t)-\delta I(t), \\
\frac{d V(t)}{d t}=p I(t)-c_{\mathrm{deg}} V(t)-\sum_{i=0}^{9} \delta_{\text {Dirac }}\left(t-t_{i}\right) \varepsilon V(t),
\end{gathered}
$$

where parameter $\varepsilon$ denotes the fraction of virus removed by the sampling (i.e., $\varepsilon=0.854$ ), and the rate of virus degradation, $c_{\mathrm{deg}}$, is only that due to degradation of either total extracellular virus (for virus quantified in RNA copies) or virus infectivity (for virus quantified in $\mathrm{TCID}_{50}$ ). In the above equation, the removal of virus due to sampling is captured explicitly by an impulsive term, unlike in Eq.(1) where $c$ also includes harvesting of the medium. As such, the value for $c$ in Eq.(1) (reported in Tables 2 and 3) is equal to the value of $c_{\mathrm{deg}}$ in the above model with punctual virus removal (reported in Tables 4 and 5) plus the rate of virus loss due to harvesting, namely $1.92 /$ day $\left(-\ln (1-0.854) /(1\right.$ day) $)$. Both the values of $c$ and $c_{\text {deg }}$ were determined in earlier work (Iwami et al., 2012a; Iwami et al., 2012b), and fixed to their previously determined value in the present work. We define $t_{i}=i$ days for $i=0,1,2, \ldots, 9$ because the experiments last from day 0 to 9 , and $\delta_{\text {Dirac }}$ is the Dirac delta such that

$$
\int_{0}^{t} \delta_{\text {Dirac }}\left(s-t_{i}\right) \varepsilon V(s) \mathrm{d} s=\sum_{i=0}^{i<t} \varepsilon V\left(t_{i}\right) .
$$

340 Similar to the derivation of Eq.(2) and Eq.(3), we have

$$
\frac{d T(t)}{d t}+\frac{d I(t)}{d t}+\frac{\delta}{p} \frac{d V(t)}{d t}-\frac{\delta c_{\mathrm{deg}}}{p \beta} \frac{d L(t)}{d t}+\frac{\delta}{p}\left(\sum_{i=0}^{9} \delta_{\text {Dirac }}\left(t-t_{i}\right) \varepsilon V(t)\right)=0,
$$

341 and integrating both side, we obtain

$$
H=T(t)+I(t)+\frac{\delta}{p} V(t)-\frac{\delta c_{\mathrm{deg}}}{p \beta} L(t)+\frac{\delta}{p}\left(\sum_{i=0}^{i<t} \varepsilon V\left(t_{i}\right)\right) .
$$

342 Therefore, we derive the following normalized, conserved quantity for the punctual removal 343 model: 


$$
Q=1=\frac{1}{T(0)}\left\{T(t)+I(t)+\frac{V(t)-V(0)}{(p / \delta)}+\frac{\delta c_{\mathrm{deg}} \int_{0}^{t} V(s) d s}{(p / \delta)}+\frac{\sum_{i=0}^{i<t} \varepsilon V\left(t_{i}\right)}{(p / \delta)}\right\}
$$

\subsection{Derivation of a general conservation law for viral infections}

The eclipse phase is the time elapsed between the successful infection of a cell and the release of the first virion released by that newly infected cell (Petravic et al., 2014; Pinilla et al.,

348 2012). Interestingly, Eq.(3) can be generalized for models which include an eclipse phase. By 349 modifying Eq.(1) to incorporate an exponentially $\left(n_{E}=1\right)$ or Erlang-distributed $\left(n_{E}>1\right)$ 350 eclipse phase with mean of $\tau_{E}$, where $n_{E}$ is the number of exponentially distributed eclipse 351 compartments in the model, and modifying $d I(t) / d t$ accordingly, one obtains the more 352 general model for $i=2 \ldots n_{E}$

$$
\begin{gathered}
\frac{d T(t)}{d t}=-\beta T(t) V(t), \\
\frac{d E_{1}(t)}{d t}=\beta T(t) V(t)-\frac{n_{E}}{\tau_{E}} E_{1}(t), \\
\frac{d E_{i}(t)}{d t}=\frac{n_{E}}{\tau_{E}} E_{i-1}(t)-\frac{n_{E}}{\tau_{E}} E_{i}(t), \\
\frac{d I(t)}{d t}=\frac{n_{E}}{\tau_{E}} E_{n_{E}}(t)-\delta I(t), \\
\frac{d V(t)}{d t}=p I(t)-c V .
\end{gathered}
$$

353 It is easy to show that the corrected quantity $H$ for this model is simply

$$
H=T(t)+\sum_{i=1}^{n_{E}} E_{i}(t)+I(t)+\frac{\delta}{p} V(t)-\frac{\delta c}{p \beta} L(t)
$$

354 which simplifies to Eq.(3) when $\sum_{i=1}^{n_{E}} E_{i}(t)=0$, i.e. when there is no eclipse phase. If one 355 wanted to compute $H$ experimentally, cells in the eclipse phase would appear either as 356 uninfected or infected cells and so the experimental calculation of $H$ would follow Eq.(1), 357 independently of the choice to use a model with or without an eclipse phase. 


\section{Acknowledgments}

This research was supported in part by the Kyushu University Fund (Y.K.), JST CREST

361 program (K.S. and S.I.), the Aihara Innovative Mathematical Modeling Project, JSPS, through

362 the "Funding Program for World-Leading Innovative R \& D on Science and Technology (FIRST

363 Program)", initiated by Council for Science and Technology Policy (to K.S. and S.I.), Takeda

364 Science Foundation (to K.S.), Sumitomo Foundation Research Grant (to K.S.), Senshin Medical

365 Research Foundation (to K.S.), Imai Memorial Trust for AIDS Research (to K.S.), Ichiro

366 Kanehara Foundation (to K.S.), Kanae Foundation for the Promotion of Medical Science (to

367 K.S.), Suzuken Memorial Foundation (to K.S.), Kyushu University Short-term Young Scholar

368 Exchange Program, which is based on Japanese Ministry of Education, Culture, Sports, Science

369 and Technology's grant The Program for Promoting the Enhancement of Research Universities

370 (C.A.A.B. and S.I.), JST PRESTO program (S.I.), Grants-in-Aid for Young Scientists

371 B25800092 from the Japan Society for the Promotion of Science or JSPS (S.I.), with additional

372 funding from the Inamori Foundation (S.I.).

\section{Authors contributions}

Conceived and designed the experiments: TM KS SI. Performed the experiments: TM.

379 Analyzed the data: YK SM SN YT CAAB SI. Contributed reagents/materials/ analysis tools:

380 TM. Wrote the paper: YK SM SN YT KS TM CAAB SI. 


\section{References}

Akari, H., Mori, K., Terao, K., Otani, I., Fukasawa, M., Mukai, R., Yoshikawa, Y., 1996. In vitro immortalization of Old World monkey T lymphocytes with Herpesvirus saimiri: its susceptibility to infection with simian immunodeficiency viruses. Virology 218, 382-8, doi:10.1006/viro.1996.0207.

Anderson, R. M., 1991. Discussion: the Kermack-McKendrick epidemic threshold theorem. Bull Math Biol 53, 3-32.

Beauchemin, C. A., McSharry, J. J., Drusano, G. L., Nguyen, J. T., Went, G. T., Ribeiro, R. M., Perelson, A. S., 2008. Modeling amantadine treatment of influenza A virus in vitro. J Theor Biol 254, 439-51, doi:10.1016/j.jtbi.2008.05.031.

Blanchard, P., Devaney, R., Hall, G., 2005. Differential Equations. Thomson Brooks/Cole, Belmont.

Clapham, P. R., Weiss, R. A., Dalgleish, A. G., Exley, M., Whitby, D., Hogg, N., 1987. Human immunodeficiency virus infection of monocytic and T-lymphocytic cells: receptor modulation and differentiation induced by phorbol ester. Virology 158, 44-51.

Fukuhara, M., Iwami, S., Sato, K., Nishimura, Y., Shimizu, H., Aihara, K., Koyanagi, Y., 2013. Quantification of the dynamics of enterovirus 71 infection by experimental-mathematical investigation. J Virol 87, 701-5, doi:10.1128/jvi.01453-12.

Hagiwara, T., Ushimaru, T., Tainaka, K., Kurachi, H., Yoshimura, J., 2011. Apoptosis at inflection point in liquid culture of budding yeasts. PLoS One 6, e19224, doi:10.1371/journal.pone.0019224.

Holder, B. P., Beauchemin, C. A., 2011. Exploring the effect of biological delays in kinetic models of influenza within a host or cell culture. BMC Public Health 11 Suppl 1, S10, doi:10.1186/1471-2458-11-s1-s10.

Iwami, S., Sato, K., De Boer, R. J., Aihara, K., Miura, T., Koyanagi, Y., 2012a. Identifying viral parameters from in vitro cell cultures. Front Microbiol 3, 319, doi:10.3389/fmicb.2012.00319.

Iwami, S., Holder, B. P., Beauchemin, C. A., Morita, S., Tada, T., Sato, K., Igarashi, T., Miura, T., 2012b. Quantification system for the viral dynamics of a highly pathogenic simian/human immunodeficiency virus based on an in vitro experiment and a mathematical model. Retrovirology 9, 18, doi:10.1186/1742-4690-9-18.

Kermack, W., McKendrick, A., 1927. A contribution to the mathematical theory of epidemics. Proc R Soc A 115, 700-21.

Kozyrev, I. L., Ibuki, K., Shimada, T., Kuwata, T., Takemura, T., Hayami, M., Miura, T., 2001. Characterization of less pathogenic infectious molecular clones derived from 
acute-pathogenic SHIV-89.6p stock virus. Virology 282, 6-13, doi:10.1006/viro.2000.0839.

Lotka, A., 1956. Elements of Physical Biology (Williams and Wilkins, Baltimore, 1925). Elements of Mathematical Biology.

Mohler, L., Flockerzi, D., Sann, H., Reichl, U., 2005. Mathematical model of influenza A virus production in large-scale microcarrier culture. Biotechnol Bioeng 90, 46-58, doi:10.1002/bit.20363.

Motohara, M., Ibuki, K., Miyake, A., Fukazawa, Y., Inaba, K., Suzuki, H., Masuda, K., Minato, N., Kawamoto, H., Nakasone, T., Honda, M., Hayami, M., Miura, T., 2006. Impaired T-cell differentiation in the thymus at the early stages of acute pathogenic chimeric simian-human immunodeficiency virus (SHIV) infection in contrast to less pathogenic SHIV infection. Microbes Infect 8, 1539-49, doi:10.1016/j.micinf.2006.01.011.

Nowak, M., May, R. M., 2000. Virus Dynamics: Mathematical Principles of Immunology and Virology: Mathematical Principles of Immunology and Virology. Oxford university press.

Perelson, A. S., 2002. Modelling viral and immune system dynamics. Nat Rev Immunol 2, 28-36, doi:10.1038/nri700.

Petravic, J., Ellenberg, P., Chan, M. L., Paukovics, G., Smyth, R. P., Mak, J., Davenport, M. P., 2014. Intracellular dynamics of HIV infection. J Virol 88, 1113-24, doi:10.1128/jvi.02038-13.

Pinilla, L. T., Holder, B. P., Abed, Y., Boivin, G., Beauchemin, C. A., 2012. The H275Y neuraminidase mutation of the pandemic $\mathrm{A} / \mathrm{H} 1 \mathrm{~N} 1$ influenza virus lengthens the eclipse phase and reduces viral output of infected cells, potentially compromising fitness in ferrets. J Virol 86, 10651-60, doi:10.1128/jvi.07244-11.

Reed, L., Muench, H., 1938. A simple method of estimating fifty per cent endpoints. Am J Hyg 27, 493-7.

Shinohara, K., Sakai, K., Ando, S., Ami, Y., Yoshino, N., Takahashi, E., Someya, K., Suzaki, Y., Nakasone, T., Sasaki, Y., Kaizu, M., Lu, Y., Honda, M., 1999. A highly pathogenic simian/human immunodeficiency virus with genetic changes in cynomolgus monkey. J Gen Virol 80 ( Pt 5), 1231-40.

Uechi, L., Akutsu, T., 2012. Conservation laws and symmetries in competitive systems. Progress of Theoretical Physics Supplement 194, 210-222.

Uechi, L., Akutsu, T., 2013. Stability and restoration phenomena in competitive systems. Progress of Theoretical and Experimental Physics 2013, 103J01. 


\section{$455 \quad$ Figure legends}

Figure 1. Model against experimental data based on the total viral load (RNA copies). vitro experimental quantity was measured daily from $t=0 \mathrm{~d}$ to $9 \mathrm{~d}$. The solid curves in (A) and (B) show the best-fit of Eq.(1) to the experimental data (points) for the target cells, infected cells, and the total viral load (RNA copies/ml) for infection with an MOI of $2.0 \times 10^{-4}$ of SHIV-KS661 and SHIV-\#64, respectively. The black circles in (C) and (D) are values of conserved quantity $Q$, computed throughout the viral infection, using parameters extracted from the fit presented in $(\mathbf{A})(\mathbf{B})$ and experimental data points from a separate infection experiment conducted at an MOI of $2.0 \times 10^{-5}$ with SHIV-KS661 and SHIV-\#64, respectively. The dotted line represents the theoretical value of $Q=1$ assuming that the conservation law holds and that no experimental errors exist in this isolated system. Panels $(\mathbf{E})(\mathbf{F})$ and $(\mathbf{G})(\mathbf{H})$ are results obtained with the model with punctual removal (Appendix 5.1.) and are analogous to

Figure 2. Model against experimental data based on the infectious viral titer (TCID $\left._{50}\right)$. vitro experimental quantity was measured daily from $t=0 \mathrm{~d}$ to $9 \mathrm{~d}$. The solid curves in (A) and (B) show the best-fit of Eq.(1) to the experimental data (points) for the target cells, infected cells, and the infectious viral titer $\left(\mathrm{TCID}_{50} / \mathrm{ml}\right)$ for infection with an MOI of $2.0 \times 10^{-4}$ of SHIV-KS661 and SHIV-\#64, respectively. The grey circles in (C) and (D) are values of conserved quantity $Q$, computed throughout the viral infection, using parameters extracted from 478 the fit presented in $(\mathbf{A})(\mathbf{B})$ and experimental data points from a separate infection experiment 479 conducted at an MOI of $2.0 \times 10^{-5}$ with SHIV-KS661 and SHIV-\#64, respectively, 480 throughout the viral infection. The dotted line represents the theoretical value of $Q=1$ 481 assuming that the conservation law holds and that no experimental errors exist in this isolated 482 system. Panels $(\mathbf{E})(\mathbf{F})$ and $(\mathbf{G})(\mathbf{H})$ are results obtained with the model with punctual removal 483 (Appendix 5.1.) and are analogous to panels (A)(B) and (C)(D), respectively. 


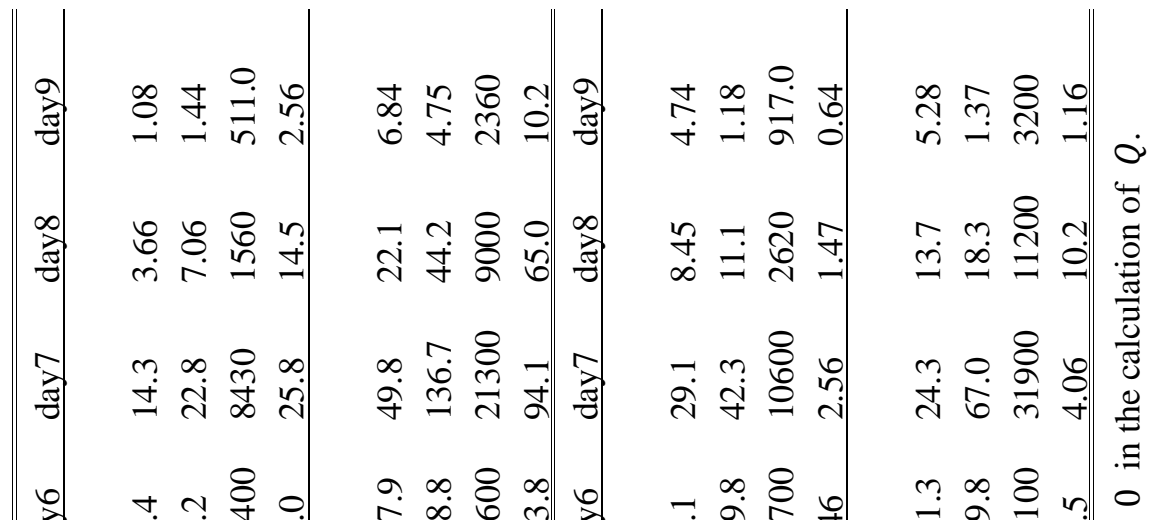

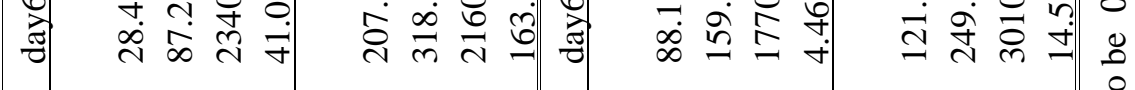

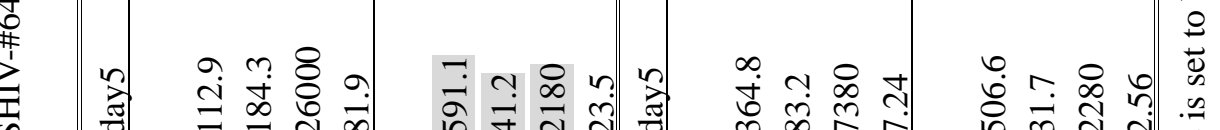

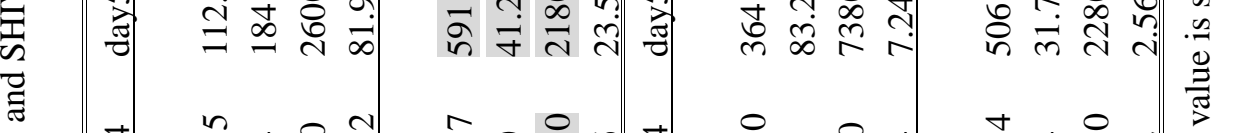

त)

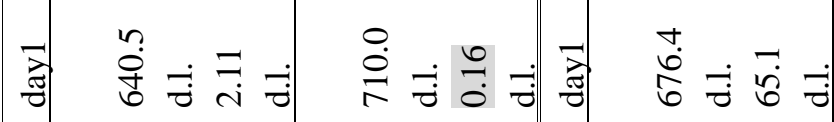

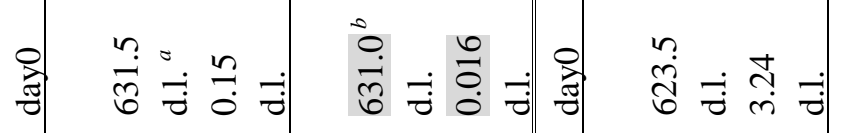

芒芦芦芦

ใิ

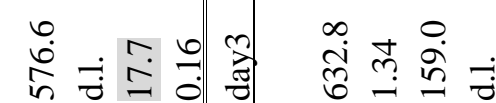

言守富

면

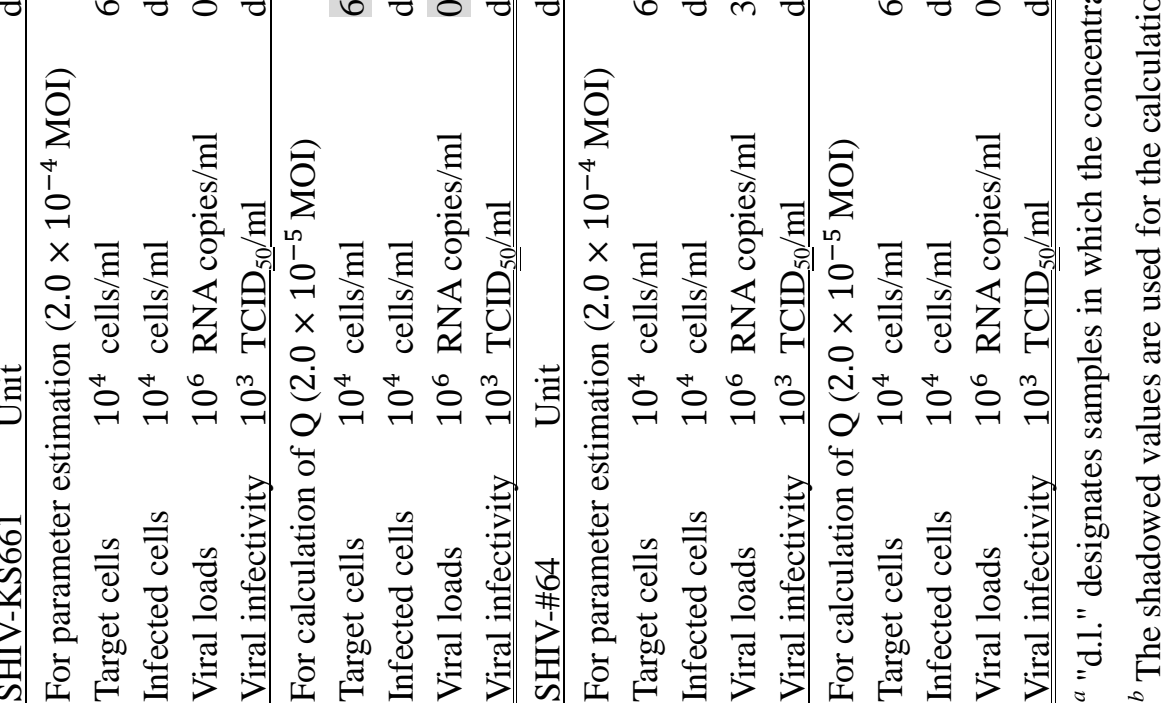




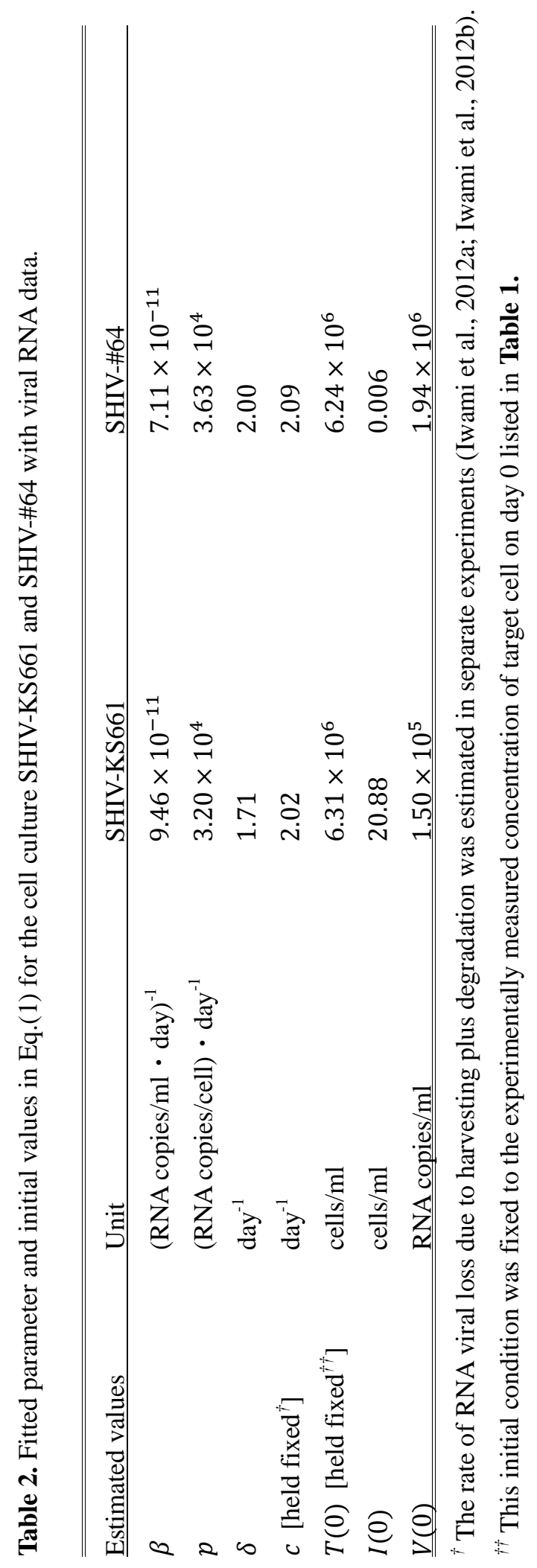




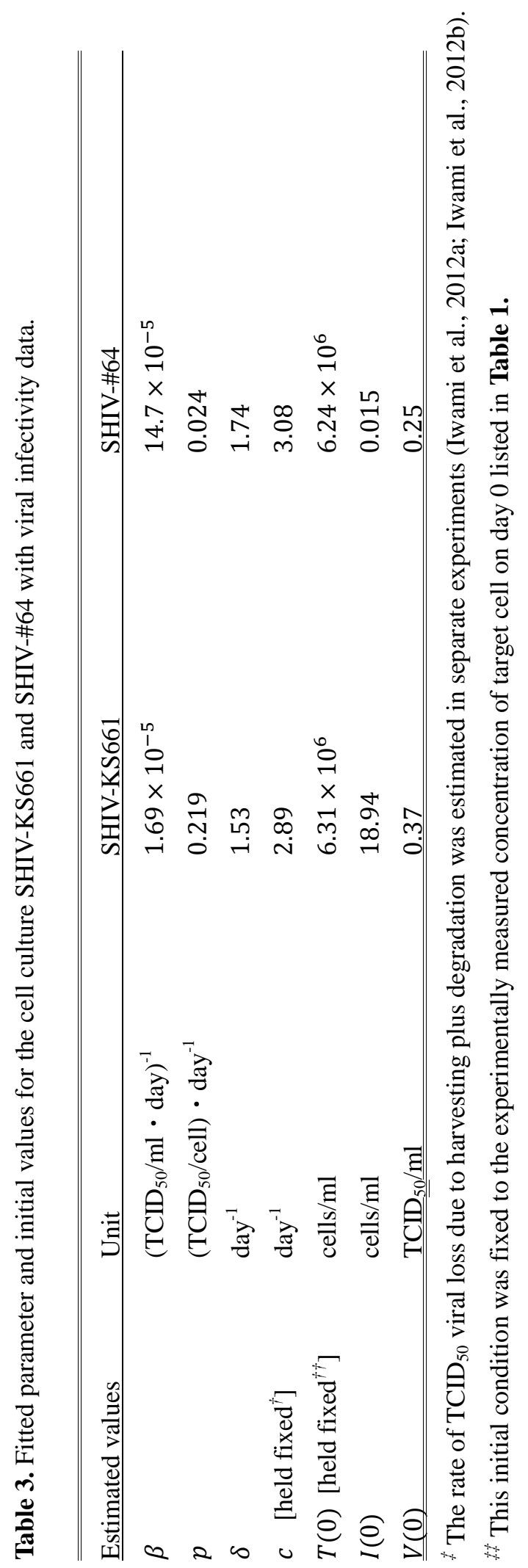




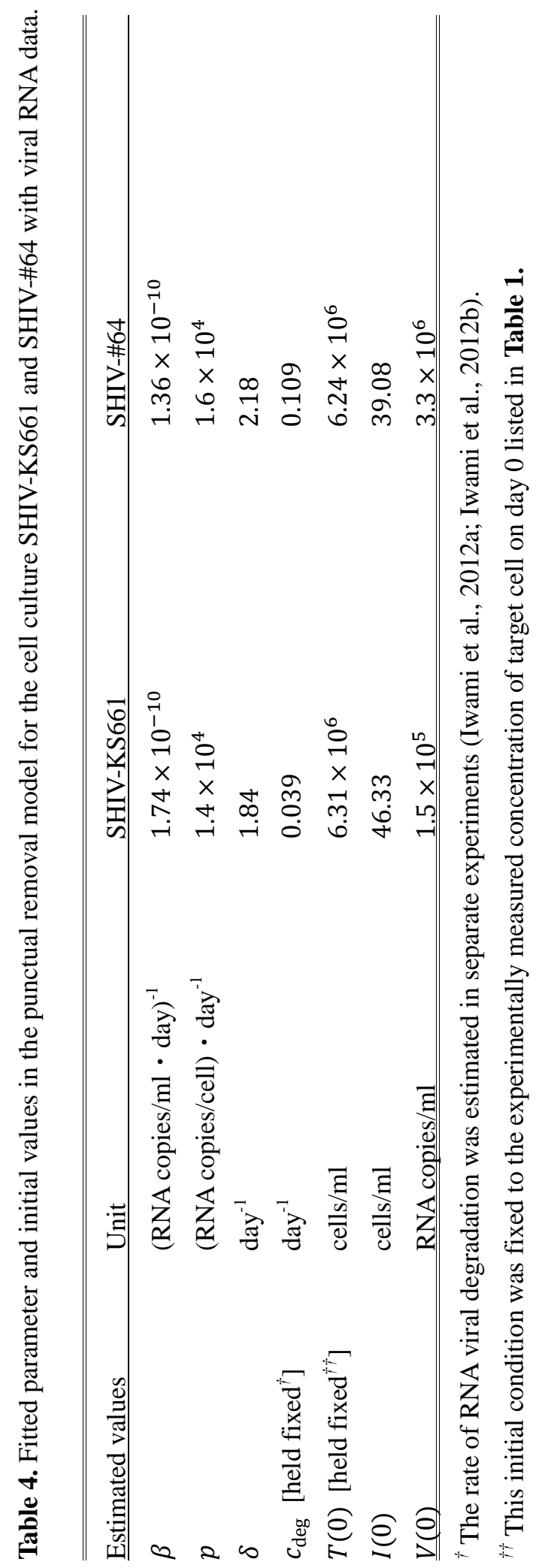




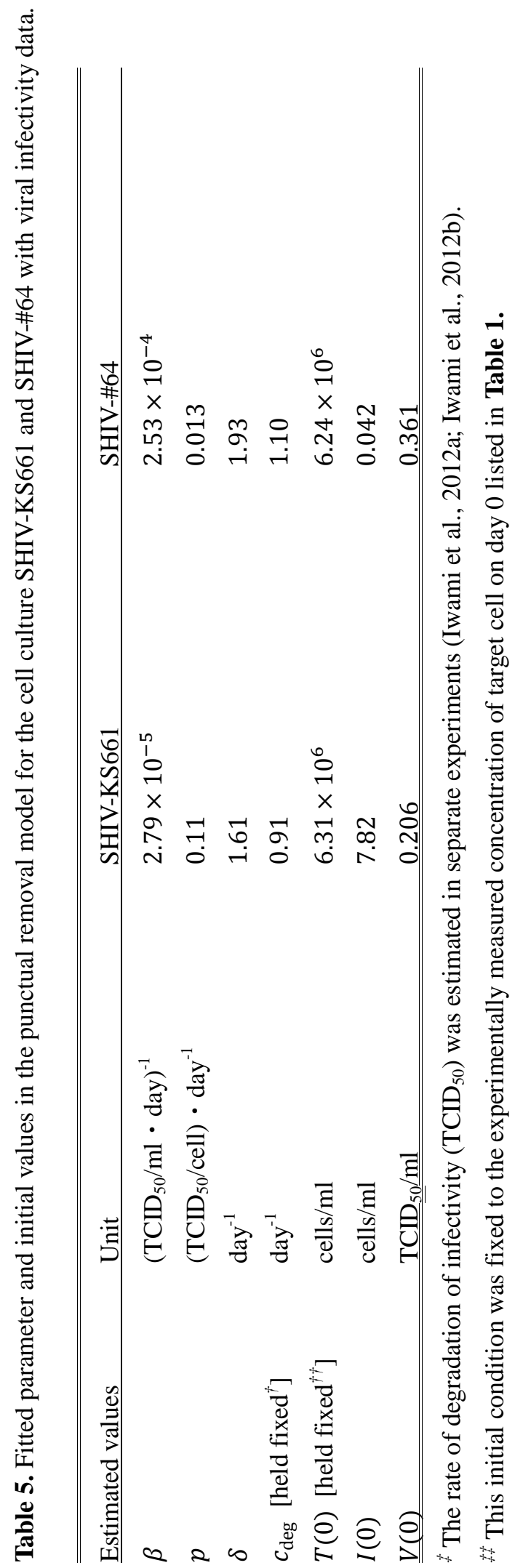




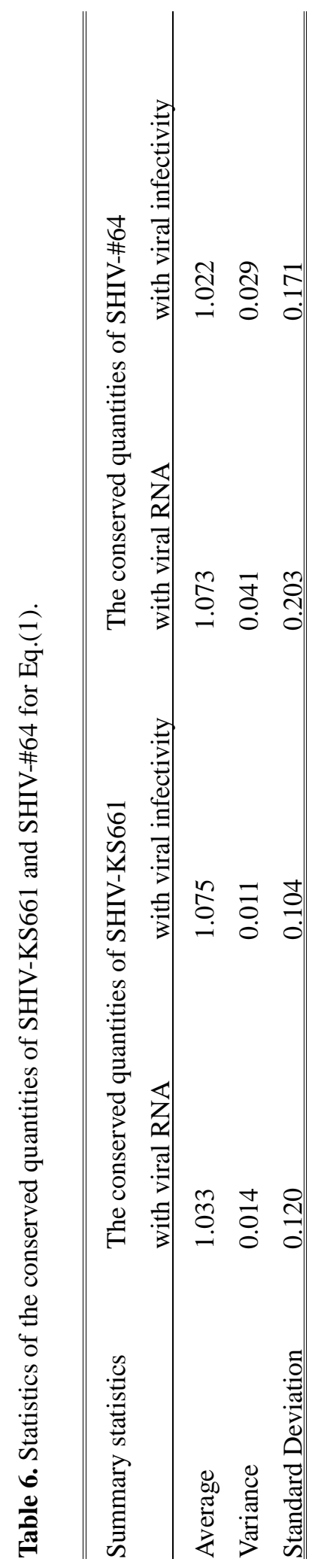




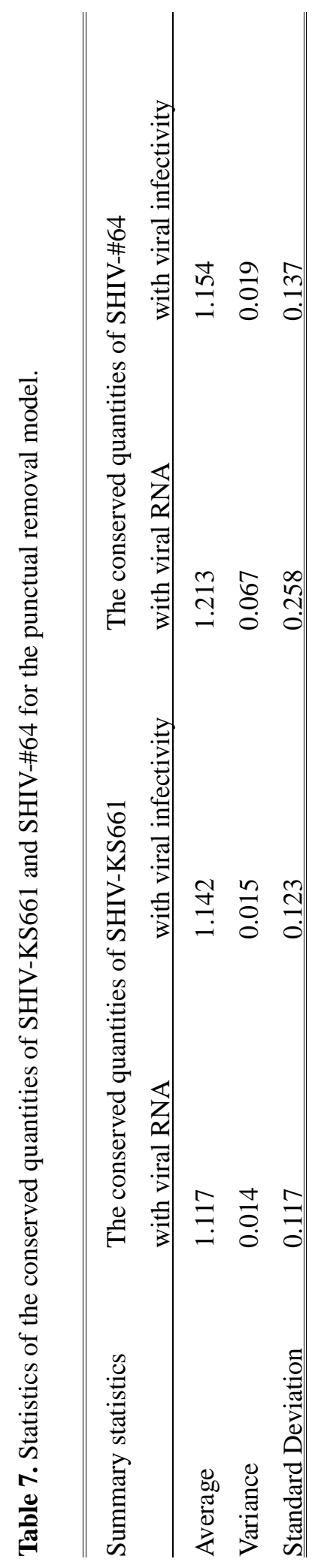


Figure 1
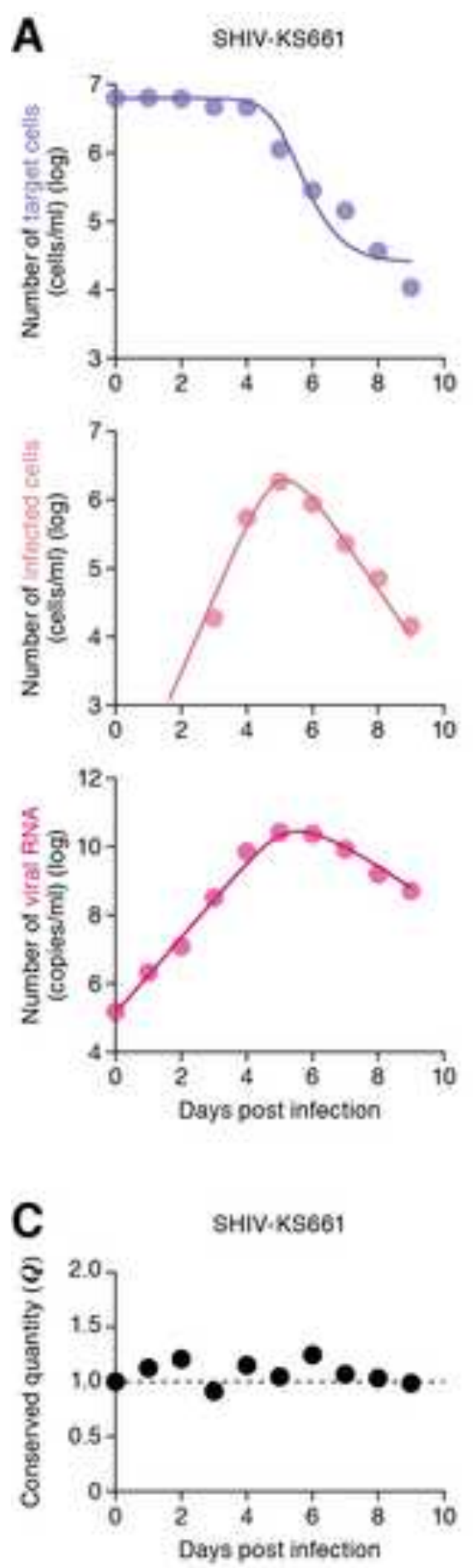
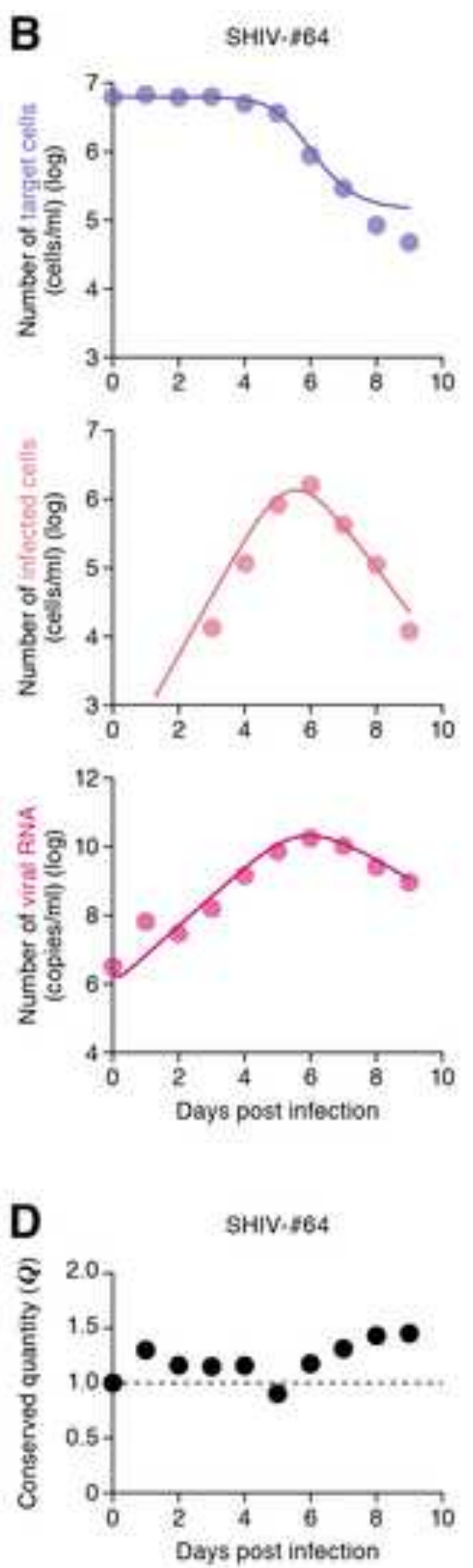
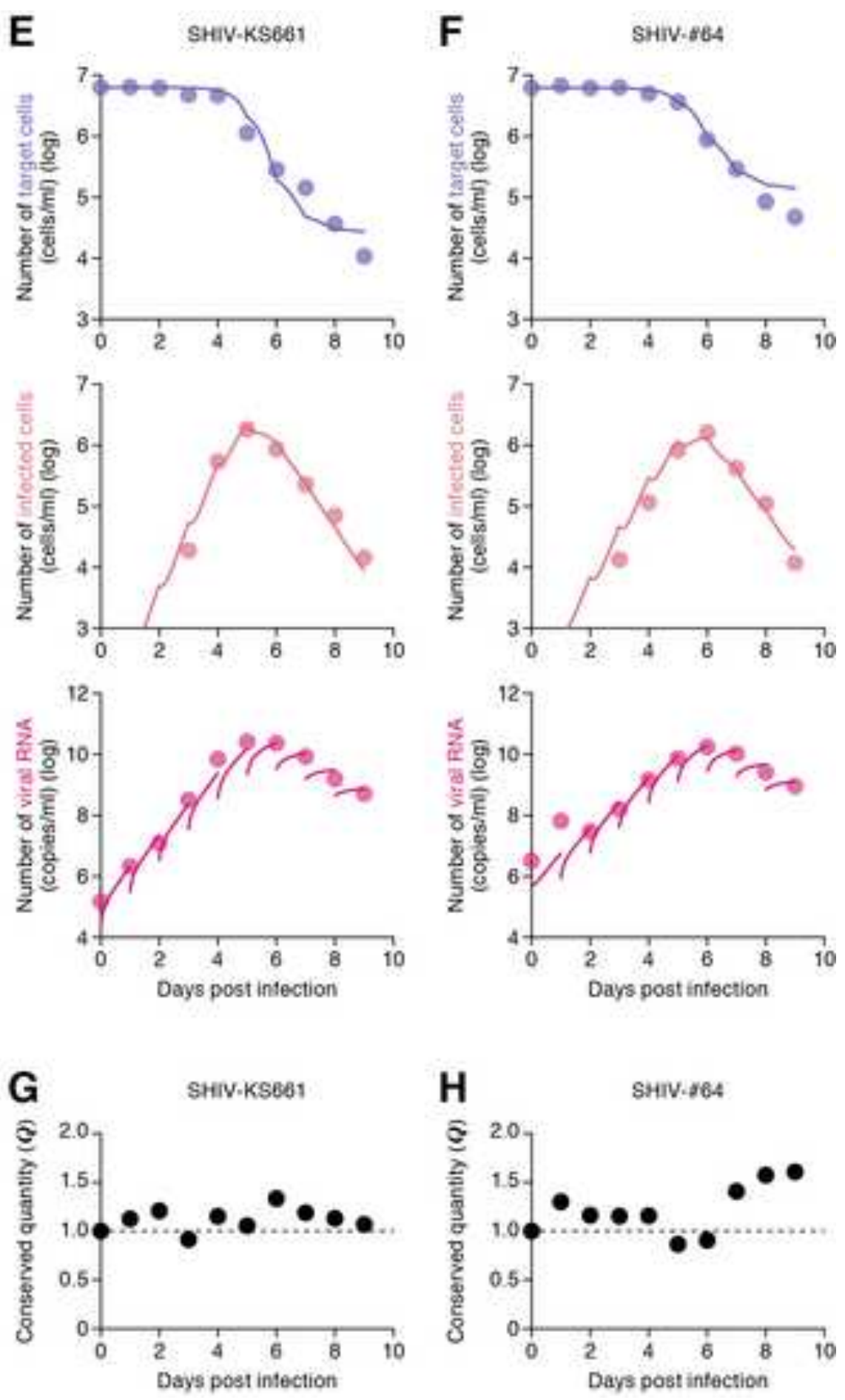
A
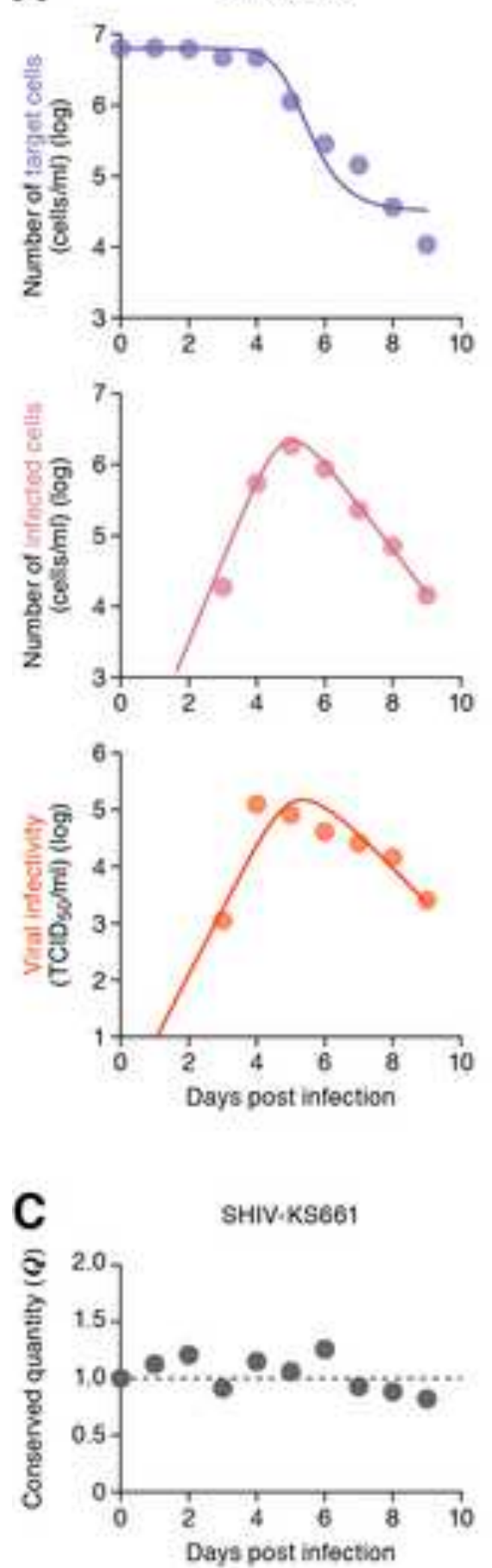

B
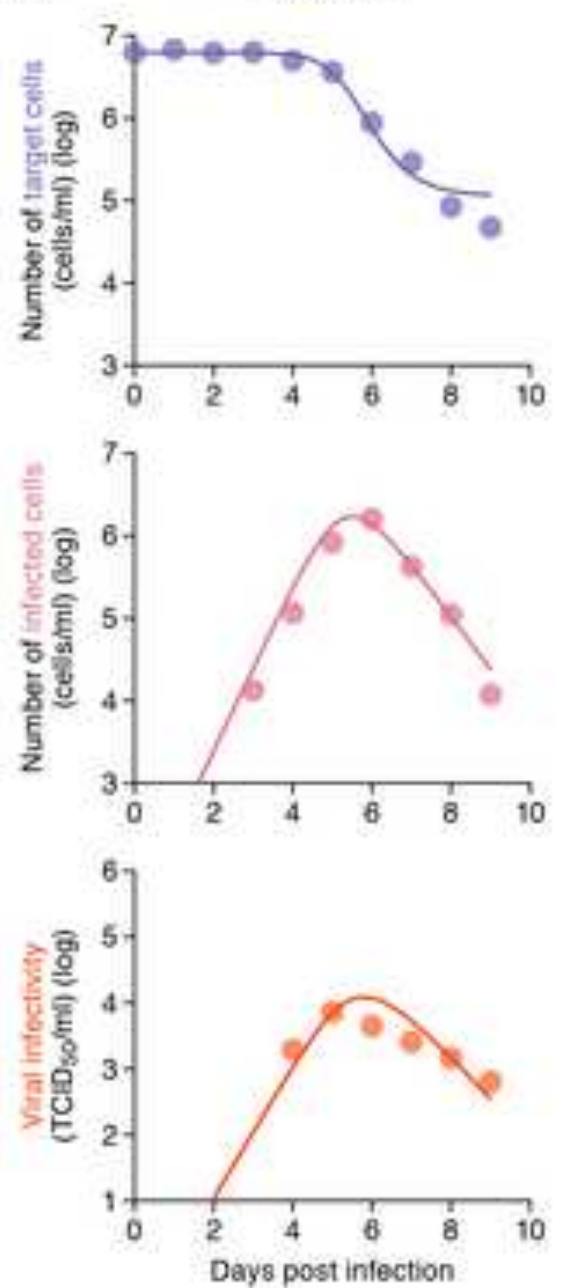

D \$HIV.\$64

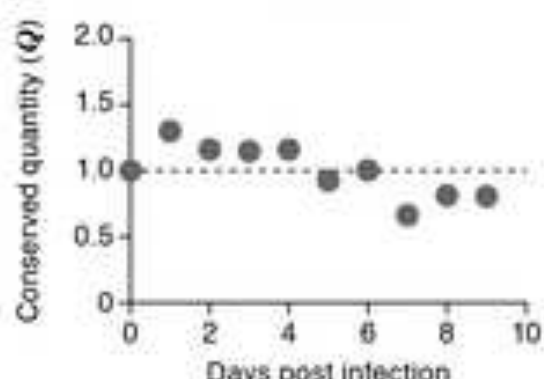

E
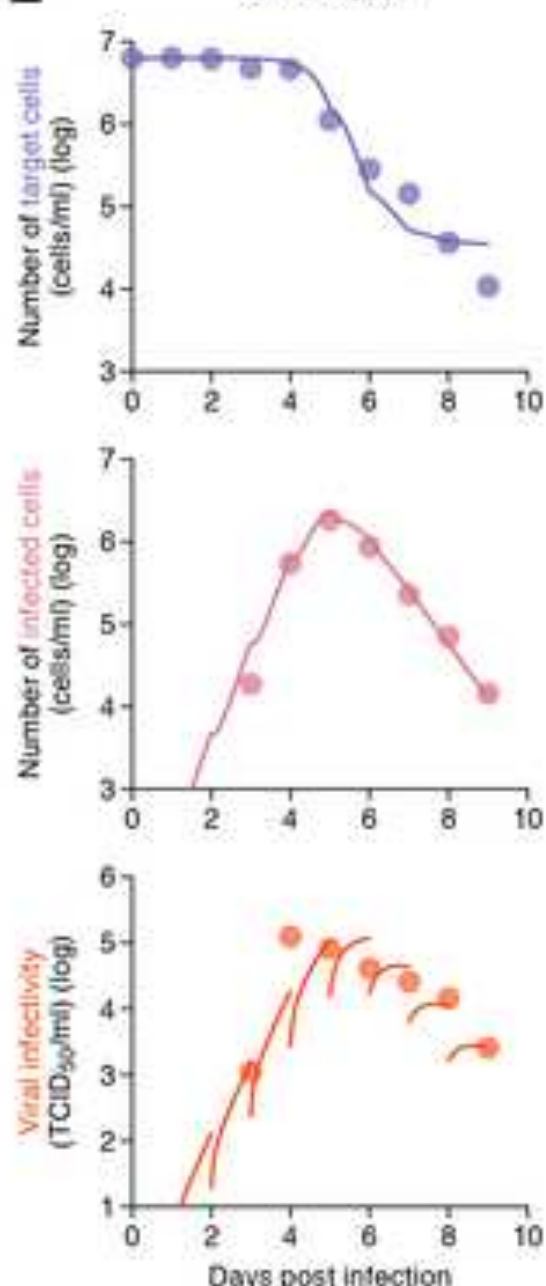

G

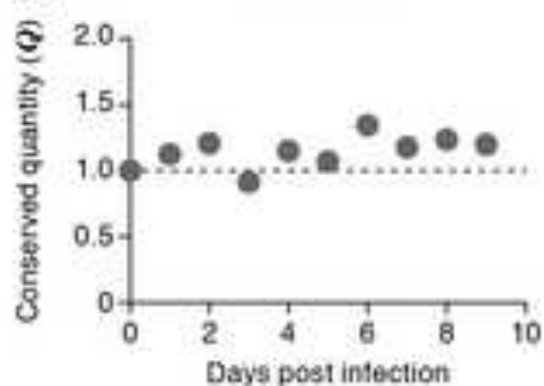

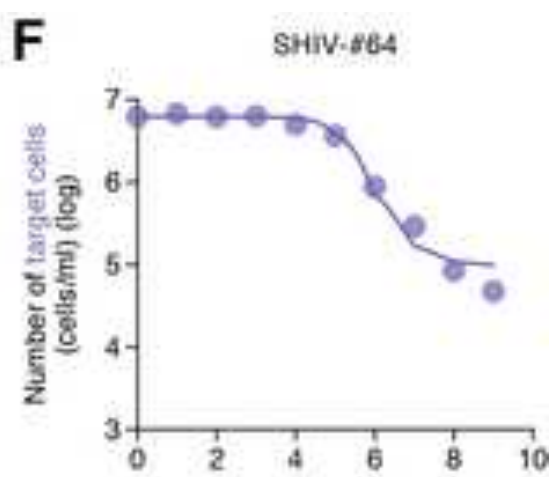
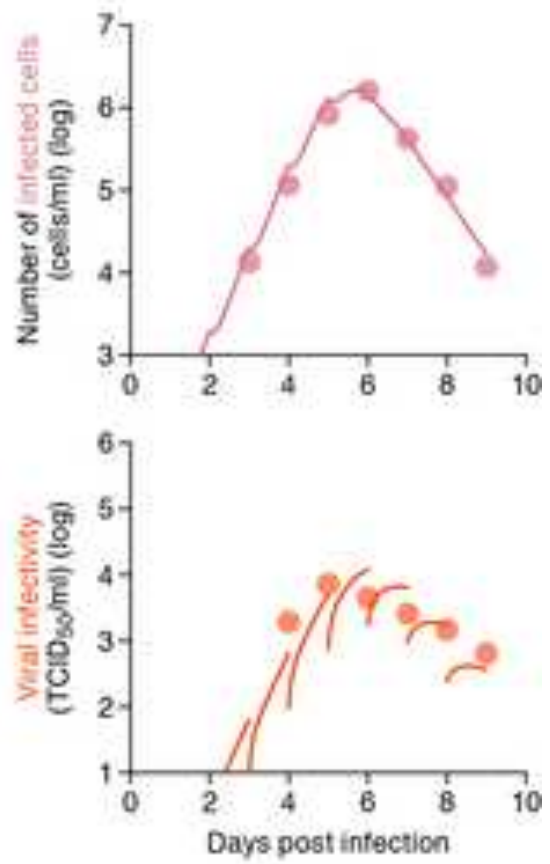

H SHIV.764

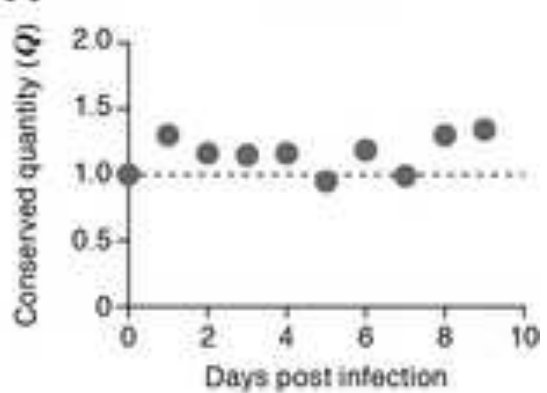

